\title{
Quality Improvement Interventions for Early HIV Infant Diagnosis in Northeastern Uganda
}

\author{
Jonathan Izudi, ${ }^{1}$ Agnes Akot, ${ }^{2}$ Grace Paul Kisitu, ${ }^{3}$ \\ Pauline Amuge, ${ }^{3}$ and Adeodata Kekitiinwa ${ }^{3}$ \\ ${ }^{1}$ Baylor College of Medicine Children's Foundation-Uganda, Mulago Regional Referral Hospital, Block-Clock Tower, \\ P.O. Box 72052, Karamoja Region, Kampala, Uganda \\ ${ }^{2}$ Kaabong District Local Government, Kaabong District Hospital, ANC Clinic, Kaabong, Uganda \\ ${ }^{3}$ Baylor College of Medicine Children's Foundation-Uganda, Clinical Center of Excellence, Mulago Regional Referral Hospital, \\ Block-Clock Tower, P.O. Box 72052, Kampala, Uganda
}

Correspondence should be addressed to Jonathan Izudi; jonahzd@gmail.com

Received 9 September 2016; Revised 22 November 2016; Accepted 5 December 2016

Academic Editor: Lucia Lopalco

Copyright (C) 2016 Jonathan Izudi et al. This is an open access article distributed under the Creative Commons Attribution License, which permits unrestricted use, distribution, and reproduction in any medium, provided the original work is properly cited.

Introduction. Early infant diagnosis (EID) of human immunodeficiency virus (HIV) ensures prompt treatment and infant survival. In Kaabong Hospital, 20\% of HIV exposed infants (HEIs) had access to HIV diagnosis by eight weeks. We aimed to improve EID of HIV by deoxyribonucleic acid-polymerase chain reaction (DNA-PCR) testing by eight weeks from 20 to $100 \%$ between June 2014 and November 2015. Method. In this quality improvement (QI) project, EID data was reviewed, gaps prioritized using theme matrix selection, root causes analyzed using fishbone tool, and improvement changes were selected using counter measures matrix but implemented using Plan-Do-Study-Act cycle. Root causes of low first DNA-PCR testing included maternal EID ignorance, absent lost mother-baby pairs (LMBP) tracking system, and no EID performance reviews. Health education, Continuous Medical Education (CMEs), and integration of laboratory and EID services were initial improvement changes used. Results. DNA-PCR testing increased from 20 to 100\% between June 2014 and July 2015 and was sustained at 100\% until February 2016. Two declines, 67\% in September 2014 and 75\% in June 2015, due to LMBP were addressed using expert clients and peer mothers, respectively. Conclusion. Formation of WIT, laboratory service integration at MBCP, and task shifting along EID cascade improved EID outcomes at 6 weeks.

\section{The Problem}

Globally, human immunodeficiency virus (HIV) exposed infants (HEIs) have delayed access to early infant diagnosis of HIV (EID) [1]. Although new HIV infections have reduced [2], efforts towards "zero-new infections" and having $80 \%$ HIV-infected infants started on antiretroviral therapy (ART) may be reversed if EID is delayed [3]. According to the World Health Organization (WHO), three in every four HEIs have delayed access to EID services leading to high HIV-related childhood deaths in Sub-Saharan Africa despite the availability of antiretroviral therapy (ART).

EID is hence a critical facilitator of early access to ART. In doing so, it ensures optimal viral suppression, reduced incidences of opportunistic infections, and improved quality of life of HIV-positive children [4]. Conversely, EID also has critical implications for infant survival. Previous studies suggested a $76 \%$ reduced risk of infant mortality within the first 3 months of life $[5,6]$ and $75 \%$ reduced HIV progression to Acquired Immunodeficiency Syndrome (AIDS) [6] with early ART initiation. Given the aggressive course of pediatric HIV with an estimated $20 \%$ infant mortality before six months [7] and 35-40\% mortality before 12 months of age $[7,8]$, the role of EID in early ART initiation cannot be underestimated [8].

Uganda adopted Option-B plus that mandates initiation of ART in HIV-positive pregnant and lactating mothers irrespective of WHO clinical staging and immune status in order to eliminate mother to child transmission of HIV (eMTCT). 
This is implemented through a provider initiated testing and counseling (PITCT). In PITCT, all pregnant mothers are tested for HIV during antenatal care (ANC) visits, labor, and delivery and postpartum mothers are tested during postnatalcare visits. Consequently, those found HIV-positive are started on ART and cotrimoxazole prophylaxis [2].

After delivery, HEIs are started on daily Nevirapine syrup adjusted according to weight and age bands until six weeks of age. At six weeks of age, daily Nevirapine syrup is stopped and daily cotrimoxazole syrup is started but adjusted over time according to weight/age bands until 18 months of age. In terms of breast feeding, HEIs are exclusively breastfed during the first six months of life; complementary feeding commences thereafter and breastfeeding ceases at 1 year $[1,2]$.

From birth to 18 months, HEIs undergo routine testing for HIV. The first test is by deoxyribonucleic acid-polymerase chain reaction (DNA-PCR) at six weeks of age, then a second DNA-PCR test is at six weeks after cessation of breastfeeding, and a final rapid HIV antibody test is at 18 months of age [9]. Should a HEI turn positive during these three HIV testing times, ART is initiated and subsequent monitoring continued concurrently with cotrimoxazole prophylaxis. For the HIV-positive pregnant or lactating mother, ART and cotrimoxazole are continued for life while monitoring virologic suppression. Despite these recommendations, EID by DNAPCR tests among HEIs within two months of birth (first DNA-PCR) in Uganda remains low at 40.2\% [10].

This has led to late ART initiation and unfavorable pediatric HIV outcomes [4]. Second, loss to follow-up of HIVpositive mothers and HEIs continues to retard EID services. According to Uganda National Pediatric HIV Support data, $70 \%$ of HEIs are either lost to follow-up or have no final HIV antibody test at 18 months [11]. To resolve these poor EID outcomes, Uganda introduced the concept of MotherBaby Care Point (MBCP), a service delivery model within the maternal child health clinic where HIV-positive mothers and HEIs are paired, cared for together, and retained until 18 months postpartum or beyond [11].

In Kaabong Hospital, substantial proportion of HEIs have delayed access to EID by first DNA-PCR test. In June 2014, EID data analysis indicated only one in every five $(20.0 \%)$ HEIs had access to EID by first DNA-PCR test. The preceding months (March-May 2014) equally had low proportion of HEIs tested by DNA-PCR at 6 weeks. Maternal and health services related factors contributed to this low first DNAPCR testing. In Uganda, all HEIs are expected to have their first DNA-PCR test between 6 and 8 weeks of age. Our aim was therefore to increase first DNA-PCR testing from 20 to 100\% between June 2014 and November 2015 using quality improvement (QI) interventions.

\section{Background}

Quality of healthcare gained huge recognition in Uganda lately $[12,13]$. Quality improvement (QI) defined as closing gaps between current and expected performance using appropriate measures as defined by standards is relatively a new concept in Uganda.
Uganda launched the first QI strategic framework in 2010. This was followed by establishment of QI committees at various levels of healthcare (national, regional, district, health subdistrict, hospitals and its departments, and health centers) [13]. The goal was to provide high quality health services and contribute to attainment of good quality of life and well-being at all levels of healthcare $[12,14]$.

Quality of healthcare is an important mediator between the six WHO building blocks of health systems strengthening (service delivery, health work force, health information, health financing, leadership and medical products, vaccines, and technologies) and health outcomes (effectiveness, efficiency, responsiveness, and social and financial risk protection) [13].

This QI project was conducted at Kaabong General Hospital in Karamoja region, Northeastern Uganda. Karamoja region (a rural and remote setting) has poor transport network and frequent intraclan and cross-border armed raids among others. The region has limited access to health services and health information and suffers from inadequate human resources for health (below average staffing levels and high turnover) to handle the increasing burden of HIV/AIDS. In the past, Karamoja region was known for its conservative cultural practices and isolation from the rest of Uganda (dual factors that accounted for low HIV prevalence). However, in recent years, HIV prevalence increased from 3.5\% in 2005 to $5.3 \%$ in 2011 due to improved security allowing free movement of people and urbanization [15].

The region has poor EID quality of care indicators. By March 2016, merely 61\% (126/206) of HEIs received an HIV test by 6-8 weeks. Similarly, $49 \%(100 / 206)$ were started on cotrimoxazole prophylaxis within 2 months of birth. However, there was marked progress in reducing the regional HIV positivity rate among HEIs from $17 \%$ in 2012 to $2 \%$ by June 2016. In contrast, $7.4 \%$ of women in Karamoja region use modern family planning (FP) methods and $20.5 \%$ have unmet FP need [16].

Kaabong district is one of the seven districts of Karamoja region and it is among the 15 bottom performing districts in Uganda. It was ranked 105th out of 112 districts in health performance indicators [14]. Kaabong Hospital located in Kaabong district is owned by the Republic of Uganda and lies approximately 709 kilometers in the Northeast of Kampala, the Capital City of Uganda. It serves 27 lower health facilities of which one is level four, five are level three, and 21 are level two health centers. In addition, it is a referral center for patients from the bordering districts (Kitgum and Kotido) and countries (Southern Sudan and Kenya) [17]. The hospital provides general medical, surgical, pediatric, obstetric, orthopedic, nursing, HIV/ART, e-MTCT, EID, TB, ophthalmic, and dental services [17].

\section{Baseline Measurement}

In June 2014, the MBCP Work Improvement Team (WIT), a departmental QI team at the ANC unit of Kaabong Hospital, abstracted EID data for the period March-June 2014 using standardized Ministry of Health $(\mathrm{MoH})$-Uganda data abstraction form. 
TABLE 1: Theme matrix selection for prioritization of EID quality of care gaps.

\begin{tabular}{lccccc}
\hline Themes & Customers & $\begin{array}{c}\text { Impact on } \\
\text { customer } \\
\text { score }(A)\end{array}$ & $\begin{array}{c}\text { Need to } \\
\text { improve } \\
\text { score }(B)\end{array}$ & $\begin{array}{c}\text { Overall score } \\
(A \times B)\end{array}$ & $\begin{array}{c}\text { Remark on } \\
\text { selection }\end{array}$ \\
\hline $\begin{array}{l}20 \% \text { first DNA-PCR } \\
\text { testing }\end{array}$ & $\begin{array}{l}\text { HEIs and } \\
\text { caregivers }\end{array}$ & 4 & 5 & 20 & Selected \\
$\begin{array}{l}\text { 55\% second } \\
\text { DNA-PCR testing }\end{array}$ & $\begin{array}{l}\text { HEIs and } \\
\text { caregivers }\end{array}$ & 2 & 5 & 10 & Not selected \\
$\begin{array}{l}\text { 75\% HIV antibody } \\
\text { testing at 18 months }\end{array}$ & $\begin{array}{l}\text { HEIs and } \\
\text { caregivers }\end{array}$ & 5 & 3 & 15 & Not selected \\
$\begin{array}{l}\text { HEIs and } \\
\text { to caregivers result given }\end{array}$ & \begin{tabular}{l} 
caregivers \\
\hline
\end{tabular} & 3 & 3 & 9 & Not selected \\
\hline
\end{tabular}

The EID data was then summarized into frequencies and percentages to intentionally identify quality of care gaps.

Of data abstracted from all eligible HEIs, major EID gaps identified were as follows: only five out of 25 (20.0\%) had first DNA-PCR tests at 6 weeks, two out of three (66.7.0\%) had second DNA-PCR test 6 weeks after cessation of breastfeeding, five out of seven (71.4\%) had HIV antibody test at 18 months, and six out of ten $(60.0 \%)$ EID results were given to caregivers/mothers. The MBCP WIT discussed these performance indicators and prioritized them for improvement using the theme matrix selection. In the theme matrix selection, performance indicators were prioritized based on the potential negative impacts on HEIs and HIV-positive lactating mother and the urgency of the need to tackle. A ranking score of 1-5 for both the potential negative impact and urgency to tackle was used. Highest score was assigned to a performance indicator that had high negative impact and high need to solve. The overall score was the product of the rank-scores of the two measures in the theme matrix selection (Table 1). The first DNA-PCR testing scored 20, second DNA-PCR testing scored 10, HIV-serological testing at 18 months scored 15, and EID result given to caregivers or mothers scored nine. So, improving first DNA-PCR testing was prioritized for QI (Table 1).

\section{Design}

First, Baylor College of Medicine Children's FoundationUganda (Baylor-Uganda) conducted a two-day QI mentorship at Kaabong general hospital during which selected healthcare workers from all departments were oriented on Uganda National QI Framework 2010/2011-2014/2015 through 1-hour Continuous Medical Education (CME) on basic QI concepts (introduction to QI, dimensions of quality, quality grid, steps in QI, principles of QI, data use in QI, QI tools for problem identification, and analysis among others). After the CME, a Hospital QI team consisting of multidisciplinary team members, clinical, nursing, laboratory, and environmental health professionals and two community representatives, was formed. At each department (Tuberculosis, ART, MBCP, laboratory, and ART), WITs were formed to address quality of health care performance gaps.

\section{Strategy}

The MBCP WIT started a QI project using four principles of QI: identification of EID quality of care gap (problem identification), analysis of the root causes of the EID gap, development of evidence based improvement changes (solutions), and the testing and implementation of developed solutions using the Plan-Do-Study-Act (PDSA) cycle [18].

5.1. Analysis of EID Quality of Care Gaps. The fishbone tool [19] was used to analyze potential root causes of low first DNA-PCR testing. During this analytical process, the WhyWhy-Why approach for each identified root cause of low first DNA-PCR testing was used to systematically identify underlying causes until no further causes were identified [19].

The root cause were summarized and categorized as maternal and health services factors. The maternal factors included lack of knowledge on EID importance and lost mother-infant pairs in the community. Conversely, health services related factors encompassed nonintegrated laboratory services for dry blood spot (DBS) sample collection at the MBCP, health provider knowledge gap on the timing, and frequency of DNA-PCR tests due to lack of CMEs and lack of routine EID data reviews due to absence of WIT (Figure 1).

\subsection{Development of Improvement Changes and Prioritization.} The WIT developed QI solutions (improvement changes) according to identified root causes. They used the counter measures matrix to prioritize improvement changes based on feasibility and effectiveness ranking scores of 1-5 (Figure 2). Highest score was assigned to an improvement change that was highly feasible and highly effective. Using this approach, priority was given to an improvement change with the highest overall score obtained by the product of feasibility and effectiveness scores [20]. Consequently, conduct of CMEs and integration of laboratory services for DBS sample collection at the $\mathrm{MBCP}$ and health education talks at the $\mathrm{MBCP}$ were initially prioritized. Afterwards, expert clients and peer mothers were introduced to address gaps in lost mother-baby pairs.

5.3. Implementation of Improvement Changes. We used the Plan-Do-Study-Act (PDSA) cycle to implement the QI 


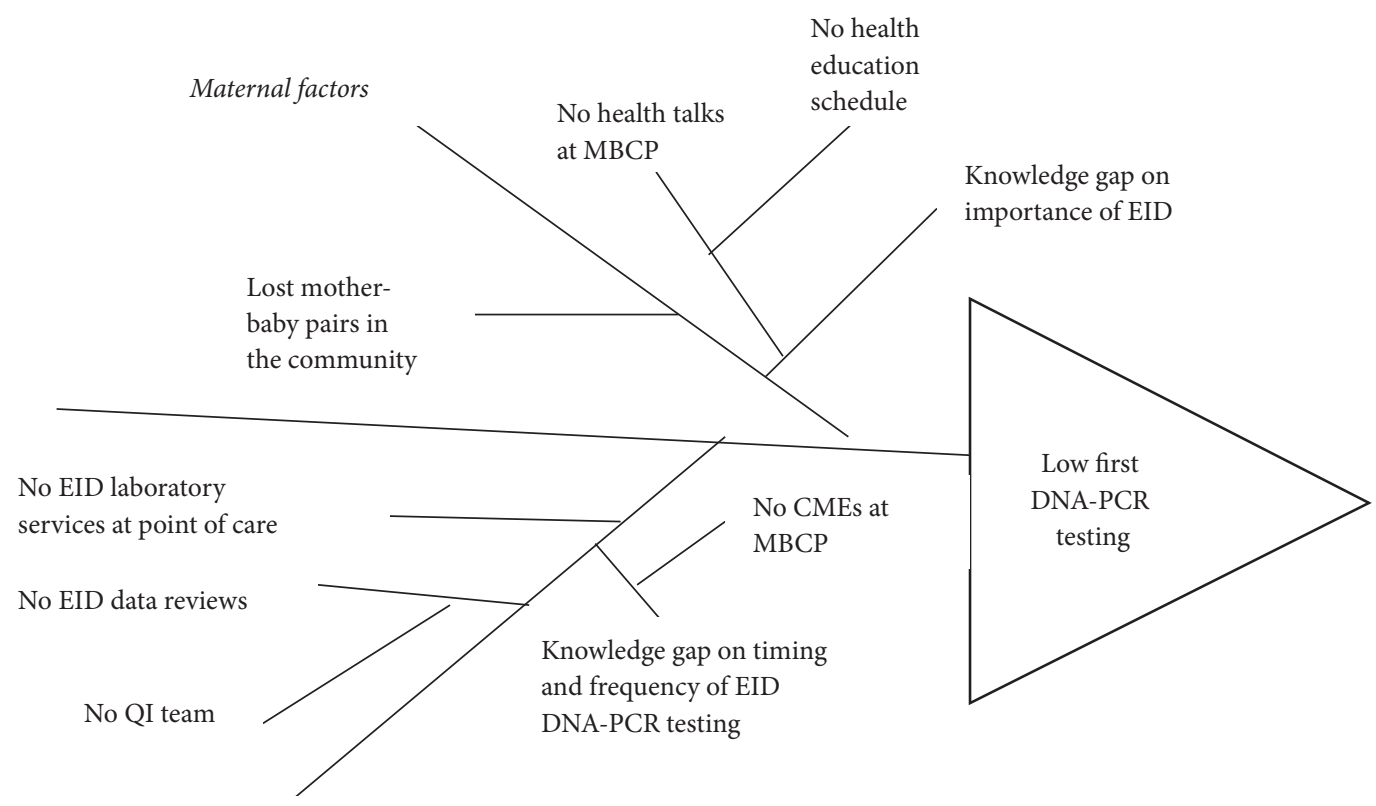

Health services factors

FIgURE 1: Fishbone analysis of the root cause of low first DNA-PCR testing.

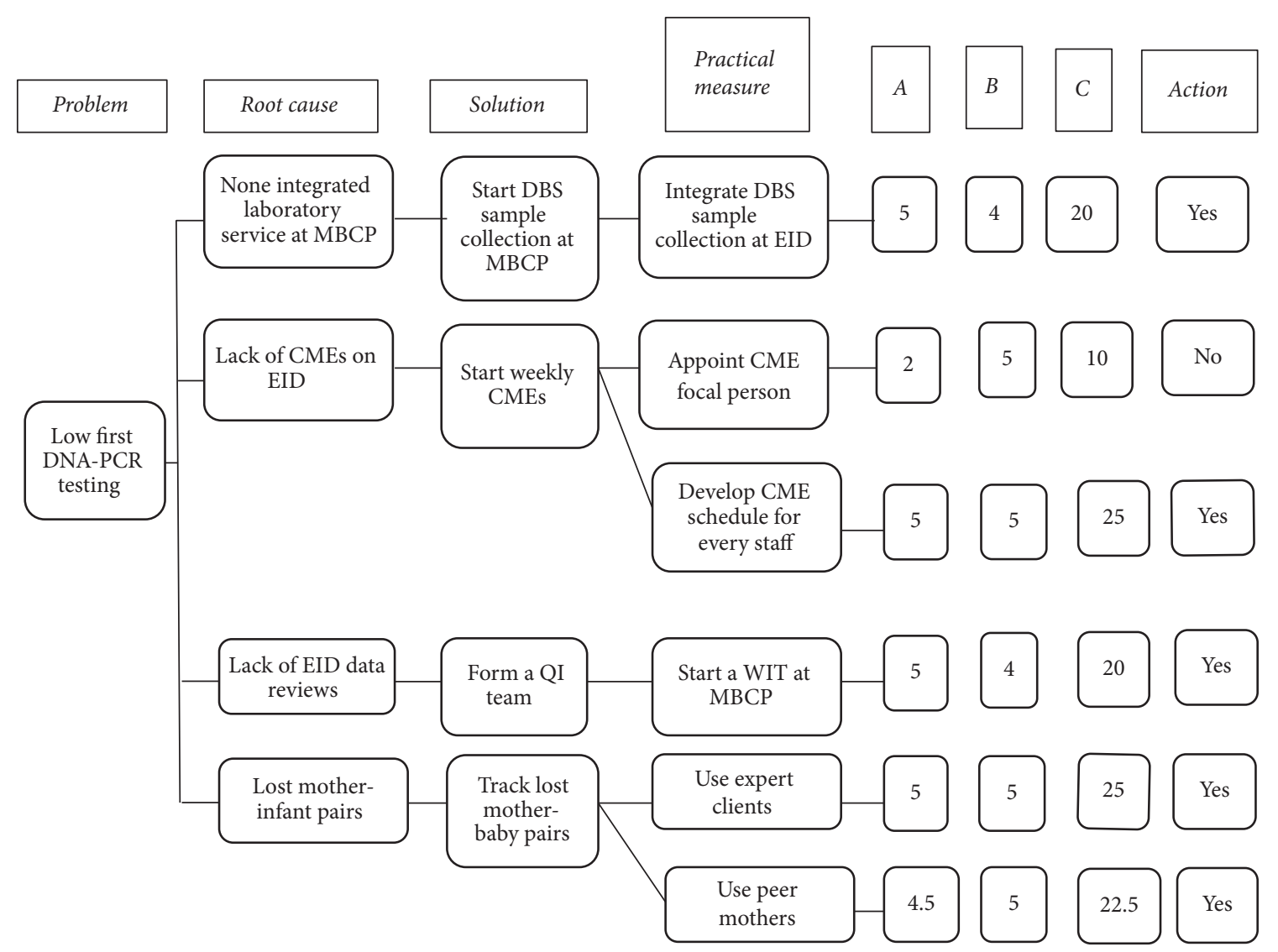

FIGURE 2: Counter measures matrix diagram showing prioritization of quality improvement changes/solutions for identified root causes. Note: $A$ : effectiveness score; $B$ : feasibility score; $C$ : product of $A$ and $B$; action: Yes = accepted and No $=$ rejected for implementation. 


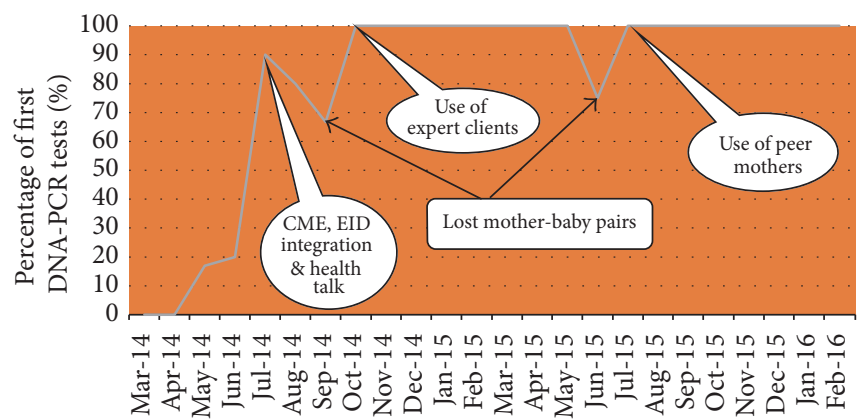

FIgURE 3: Trend of first (6-8 weeks) DNA-PCR testing among HIV exposed infants at Kaabong Hospital.

project [21]. The PDSA Cycle ensured a step-by-step implementation, monitoring and evaluation, and formulation of new QI changes or modification of current changes [22]. During implementation, a QI change that never yielded an increase in the performance indicator was dropped and new change(s) developed or existing ones were modified.

5.4. Documentation of QI Process. A QI journal consisting of start and end dates of the QI project, improvement objective, indicator, problem statement, QI solutions (changes), a line graph with annotations, and $\mathrm{f}$ lessons learnt was used to document the QI process. The set QI objective was to increase the testing of HEIs at six weeks of age by DNA-PCR from twenty percent to hundred percent between June 2014 and November 2015. The indicator was the percentage of HEIs with a DNA-PCR test within eight weeks of age. In our indicator, the numerator was the number of HEIs between 6 and 8 weeks with a documented DNA-PCR test result and the denominator was the number of all HEIs 6-8 weeks of age at the MBCP, Kaabong Hospital.

On monthly basis, the MBCP QI focal person reviewed EID clinical charts, abstracted data on first DNA-PCR testing, computed frequencies and percentages, and updated the documentation journal. EID performance indicators were shared with the rest of the WIT members at monthly QI meetings.

\section{Results}

Of eligible HEIs for first DNA-PCR (6-8 weeks) testing, testing of HEIs improved from five out of 25 (20.0\%) in June 2014 to nine out of 10 (90\%) in July 2014 when CMEs for healthcare workers and health education talks to caregivers on the importance of EID and DBS sample collection were integrated at the MBCP. However, two declines in first DNAPCR testing, two out of three (67.0\%) in September 2014 and three out of four $(75.0 \%)$ in June 2015, were recorded.

The initial decline in September 2014 was due to loss of mother-baby pairs in the community and this was addressed by use of expert clients to track lost mother-infant baby pairs in the community. The second decline in June 2015 was due to repeat loss of mother-baby pairs in the community as well and was tackled by use of peer mothers to track lost mother-infant baby pairs in the communities
(Figure 3). Other than these declines, the overall first DNAPCR testing was sustained at 100\% between June 2014 and January 2016 (Supplementary Material S1 available online at http://dx.doi.org/10.1155/2016/5625364).

\section{Lessons Learnt}

This QI project improved EID by first DNA-PCR testing at Kaabong Hospital. The introduction of CMEs, point of care DBS sample collection through integration of EID laboratory services at the MBCP, patient health education, and use of expert clients and peer mothers led to significant improvements in first DNA-PCR testing. The integration of laboratory services at the MBCP increased first DNA-PCR testing. Earlier, WHO advised health facilities to conduct onsite DBS sample collection for DNA-PCR testing in order to scale up access and coverage of PMTCT and follow-up services [23].

Our EID results obtained by point of care DBS sample collection was similar to those obtained by conventional methods. In Tanzania, similar results were reported [24]. In Kwazulu-Natal, South Africa, the integration of EID services at an immunization clinic was highly accepted by caregivers and led to increased access to EID services by HEIs at six weeks of age [25]. Similarly, in this QI project, caregivers embraced the approach and reported it to be time saving and convenient.

So onsite mentorship is essential in improving coverage and quality of EID services. In agreement with past results from Botswana, training of healthcare workers on EID DBS sample collection improved EID quality of care indicators [26].

To us, health facility based CMEs and patient health education were critical interventions. CMEs kept health workers up-to-date with EID clinical knowledge. Provision of adequate health education and counseling to caregivers on importance of EID improved self-efficacy in health matters. Health facilities providing EID services must enforce CMEs to enhance healthcare worker knowledge and improve EID quality of care. We suggest the inclusion of CME attendances as a performance appraisal indicator to improve compliance.

Identification of HEIs remains the first critical step in providing EID services and linkage to care [27]. In Mozambique, $75 \%$ of HIV-infected women never returned HEIs for EID services [8]. One of the biggest challenges to EID success in 
our setting was the high loss to follow-up. We tackled this challenge by use of expert clients and peer mothers. In the past, Uganda introduced expert clients for scaling up ART access, particularly, to ensure greater involvement of persons with HIV and to address human resource crisis [28]. In our intervention, an expert client was an HIV-positive adult with demonstrated substantial compliance to medical advice and improved clinical, physical, and immunological response to ART. He or she was willing to engage in supporting the EID program and community members for improved service delivery and is viewed as a role model by other HIV-positive persons.

Expert clients are therefore HIV-positive persons viewed by people living with HIV as motivating, influential, acceptable, appropriate, trustworthy, respectable, and morally upright to handle health matters. They voluntarily and routinely participate in health facility activities such as triaging, patient recording, health education, and counseling of difficult clients among others. In this intervention, expert clients used lost mother-baby pair sociodemographic details (village, parish, and subcounty) provided by the MBCP Focal Persons to confidentially track the pairs in the community. Upon identification, the mothers were counseled on importance of DNA-PCR tests by expert clients while in the community and physically linked to the MBCP for DNA-PCR testing.

To our surprise, the use of expert clients to track lost mother-infant pairs from the communities and their linkage to the MBCP resulted in substantial increase in DNAPCR testing because they were accessible, appropriate, and acceptable by HIV-positive mothers.

Also, Uganda introduced the concept of Family Support Groups (FSGs) as a pathway to extend HIV care, treatment, and support to family members of HIV-positive pregnant and lactating mothers. FSGs consist of HIV-positive pregnant and lactating mothers, HIV-positive children under five years, spouses to HIV-positive pregnant or lactating mothers, pregnant and lactating mothers in serodiscordant relationships, and caregivers of under 5-year-old HIV-positive children. FSGs are vital in reducing stigma at community level, offering peer support, improving group confidence, and fighting external stigma [29]. Because they are embedded in the communities, they play a great role in community engagements and this forms a critical catalyst in elimination of mother to child transmission (e-MTCT) of HIV success. Peer support practices improve demand for e-MTCT services, generate local solutions, and drive community sociobehavioral change [30].

In our setting, peer mothers were groups of women created from HIV-positive mothers within FSGs but with a HIV-negative child. This proves their compliance to eMTCT services and ability to be change agents. It is therefore not surprising that the use of peer mothers equally led to improved first DNA-PCR testing. Peer mothers were reported to be friendly, approachable, and open minded with clear EID messages compared to health workers and expert clients. To us, these acceptability differences are attributable to past experiences of peer mothers in the context of e-MTCT. Peer mothers experienced similar circumstances currently confronting HIV-positive lactating mothers in caring for
HEIs compared to expert clients. They are therefore more likely to package clearer and specific HIV messages than expert clients and healthcare workers.

\section{Conclusion}

The integration of EID laboratory services at MBCP, patient health education on importance of EID, regular EID CMEs for health workers, and use of community structures (expert clients and peer mothers) significantly improved EID outcomes and quality.

\section{Recommendation}

We recommend DBS sample collection in EID clinics, regular EID CMEs to health workers, and health education talks to HIV-positive lactating mothers in improving EID quality of care indictors. In addition, expert clients and peer mothers are very useful in improving EID coverage and outcomes. However, we noted that most health facilities in Karamoja region neglect EID. Our experience calls for active implementation of QI and adoption of these QI changes by both the public and the private healthcare settings to better EID quality of care. We strongly advice adherence to routine EID data reviews, analysis of EID quality of care gaps, generation of evidence based solutions, and multidisciplinary team composition for success.

Overall, to improve quality of health care, stronger measures in supportive supervision by multidisciplinary teams, staff motivation, continuous learning, adherence to professional code of conduct, improved logistical support to ensure adequate supplies of drugs/diagnostics, and timely payment of staff stipends when indicated are very central.

\section{Limitations}

Our QI initiative was the first systematically conducted and rigorous project in Karamoja region, Northeast Uganda. We experienced major challenges in missing EID data. In addition, the lack of qualitative information from HIV-positive lactating mothers to explain dynamics and lived experiences in EID service utilization may be limiting. Secondly, the low numbers of HEIs resulted in large variations in percentages. However, these are the total numbers of all eligible HEIs for DNA-PCR test at 6 weeks.

It was not possible to have numbers larger than those observed. In our setting, there are fewer HEIs because of low HIV prevalence in general. Also, stigma and discrimination of HIV-positive persons hinder access to and continuation with HIV care among HIV-positive mothers. The combination of these factors accounts for the low numbers of HEIs in care.

Despite these limitations, this QI project proved that simple, cost-effective, and evidence-oriented approaches can close gaps between current and expected performances as defined by standards. 


\section{Abbreviations}

AIDS: Acquired Immunodeficiency Syndrome

ART: Antiretroviral therapy

CME: Continuous Medical Education

DNA: Deoxyribonucleic acid

EID: $\quad$ Early infant diagnosis

E-MTCT: Elimination of mother to child transmission of HIV

FSGs: $\quad$ Family Support Groups

HAART: Highly active antiretroviral therapy

HEI: $\quad$ HIV exposed infants

HIV: Human immunodeficiency virus

MBCP: Mother-baby care point

PCR: $\quad$ Polymerase chain reaction

PMTCT: Prevention of mother to child transmission of HIV

POC: $\quad$ Point of care

QI: Quality improvement

WHO: World Health Organization

WIT: Work improvement team.

\section{Consent}

All participants enrolled in HIV care at Kaabong Hospital gave written informed consent prior to enrollment in HIV care.

\section{Competing Interests}

The authors declare no competing interests.

\section{Authors' Contributions}

Jonathan Izudi and Agnes Akot initiated, monitored, and evaluated the QI project. Jonathan Izudi, Agnes Akot, Pauline Amuge, Grace Paul Kisitu, and Agnes Akot equally contributed in writing this manuscript. Jonathan Izudi is first author and Agnes Akot, Pauline Amuge, Grace Paul Kisitu, Grace Paul Kisitu, and Adeodata Kekitiinwa are co-authors.

\section{Acknowledgments}

The authors recognize the great quality improvement leaderships of Dr. Andrew Katawera and Dr. Calvin Epidu, the regional laboratory quality management support by Solomon Okot Paul, and countless support from Kaabong District Health Office by Dr. Nalibe Shalif.

\section{References}

[1] E. T. Katabira, M. R. Kamya, I. Kalyesubula, and A. Namale, National Antiretroviral Treatment Guidelines for Adults, Adolescents, and Children, Ministry of Health, Kampala, Uganda, 2009.

[2] E. T. Katabira, Ed., The Integrated National Guidelines for Antiretroviral Therapy, Prevention of Mother to Child Transmission of HIV and Infant \& Young Child Feeding, Ministry of Health-Uganda, Kampala, Uganda, 1st edition, 2011.
[3] Joint United Nations Program on HIV/AIDS (UNAIDS), 9090-90 An Ambitious Treatment Target to Help End the AIDS Epidemic, WHO \& UNAIDS, Geneva, Switzerland, 2014.

[4] A. S. Hassan, E. M. Sakwa, H. M. Nabwera et al., "Dynamics and constraints of early infant diagnosis of HIV infection in rural Kenya," AIDS and Behavior, vol. 16, no. 1, pp. 5-12, 2012.

[5] C. Motswere-Chirwa, A. Voetsch, L. Lu et al., "Follow-up of infants diagnosed with HIV-early infant diagnosis program, Francistown, Botswana, 2005-2012," Morbidity and Mortality Weekly Report, vol. 63, no. 7, pp. 158-160, 2014.

[6] A. Violari, M. F. Cotton, D. M. Gibb et al., "Early antiretroviral therapy and mortality among HIV-infected infants," The New England Journal of Medicine, vol. 359, no. 21, pp. 2233-2244, 2008.

[7] A. H. Mirkuzie, S. Hinderaker, M. Sisay, K. Moland, and O. Mørkve, "Current status of medication adherence and infant follow up in the prevention of mother to child HIV transmission programme in Addis Ababa: a cohort study," Journal of the International AIDS Society, vol. 14, no. 1, article 50, 2011.

[8] R. E. Cook, P. J. Ciampa, M. Sidat et al., "Predictors of successful early infant diagnosis of HIV in a rural district hospital in Zambézia, Mozambique," Journal of Acquired Immune Deficiency Syndromes, vol. 56, no. 4, pp. e104-e109, 2011.

[9] Ministry of Health-Uganda, "The 2014 integrated guidelines for HIV/AIDS management," in Kampala AIDS Control Program, pp. 45-47, 2014.

[10] Ministry of Health-Uganda, Uganda HIV/AIDS Country Progress Report, Ministry of Health, Kampala, Uganda, 2014.

[11] AIDS Treatment and Information Center, Infectious Diseases Institute, and Makerere University, "AIDS Treatment Information Center newsletter," in Quarterly Newsletter of the AIDS Treatment Information Center, Infectious Diseases Institute, Makerere University, vol. 11, 1, 3, 5, AIDS Treatment Information Center, Infectious Diseases Institute, Makerere University, Kampala, Uganda, 2014.

[12] The Republic of Uganda, The Quality Improvement Methods: A Mannual for Health Workers in Uganda, The Republic of Uganda, Kampala, Uganda, 2015.

[13] The Republic of Uganda, Health Sector Quality Improvement Framework and Strategic Plan 2010/2011-2014/2015, vol. 9, The Republic of Uganda, Kampala, Uganda, 2011.

[14] The Republic of Uganda, Annual Health Sector Performance Report for Financial Year 2014/2015, Ministry of Health-Uganda, Kampala, Uganda, 2015.

[15] Uganda AIDS Commission, National HIV Prevention Strategy 2011-2015: Expanding and Doing HIV Prevention Better, AIDS Commission, Kampala, Uganda, 2011.

[16] Republic of Uganda, The State of Uganda Population Report 2014: Harnessing Uganda's Demographic Dividend for Socioeconomic Transformation, Republic of Uganda, Kampala, Uganda, 2014.

[17] Kaabong general hospital profile, http://www.sustainuganda .org/content/kaabong-general-hospital-profile.

[18] Ministry of Health Uganda, A Competence Based Training Manual for Health Workers in the Management of Tuberculosis in Children, National TB Leprosy Program, Kampala, Uganda, 2015.

[19] Fish bone template diagram in excel, https://www.qimacros .com/fishbone-diagram-template/.

[20] Counter Measures Matrix, https://www.qimacros.com/qualitytools/solution/. 
[21] P. Walley and B. Gowland, "Completing the circle: from PD to PDSA," International Journal of Health Care Quality Assurance, vol. 17, no. 6, pp. 349-358, 2004.

[22] Minnesota Department of Health, PDSA: Plan-Do-Study-Act, 2014.

[23] World Health Organization, WHO Recommendations on the Diagnosis of HIV Infection in Infants and Children, 2010.

[24] S. M. Lofgren, A. B. Morrissey, C. C. Chevallier et al., "Evaluation of a dried blood spot HIV-1 RNA program for early infant diagnosis and viral load monitoring at rural and remote healthcare facilities," AIDS, vol. 23, no. 18, pp. 2459-2466, 2009.

[25] N. Rollins, S. Mzolo, T. Moodley, T. Esterhuizen, and H. van Rooyen, "Universal HIV testing of infants at immunization clinics: an acceptable and feasible approach for early infant diagnosis in high HIV prevalence settings," AIDS, vol. 23, no. 14, pp. 1851-1857, 2009.

[26] T. Creek, A. Tanuri, M. Smith et al., "Early diagnosis of human immunodeficiency virus in infants using polymerase chain reaction on dried blood spots in Botswana's national program for prevention of mother-to-child transmission," The Pediatric Infectious Disease Journal, vol. 27, no. 1, pp. 22-26, 2008.

[27] S. A. Woldesenbet, D. Jackson, A. E. Goga et al., "Missed opportunities for early infant HIV diagnosis: results of a national study in South Africa," Journal of Acquired Immune Deficiency Syndromes, vol. 68, no. 3, pp. e26-e32, 2015.

[28] P. Brown-Peterside, C. A. Redding, L. Ren, and B. A. Koblin, "Acceptability of a stage-matched expert system intervention to increase condom use among women at high risk of HIV infection in New York City," AIDS Education and Prevention, vol. 12, no. 2, pp. 171-181, 2000.

[29] G. Mburu, M. Ram, M. Skovdal et al., "Resisting and challenging stigma in Uganda: the role of support groups of people living with HIV," Journal of the International AIDS Society, vol. 16, no. 3, Article ID 18636, 2013.

[30] L. A. Gulaid and K. Kiragu, "Lessons learnt from promising practices in community engagement for the elimination of new HIV infections in children by 2015 and keeping their mothers alive: summary of a desk review," Journal of the International AIDS Society, vol. 15, supplement 2, Article ID 17390, 2012. 


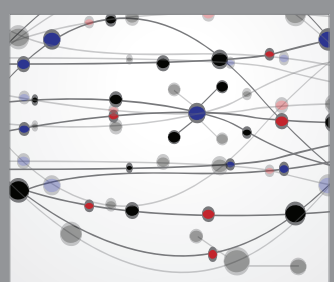

The Scientific World Journal
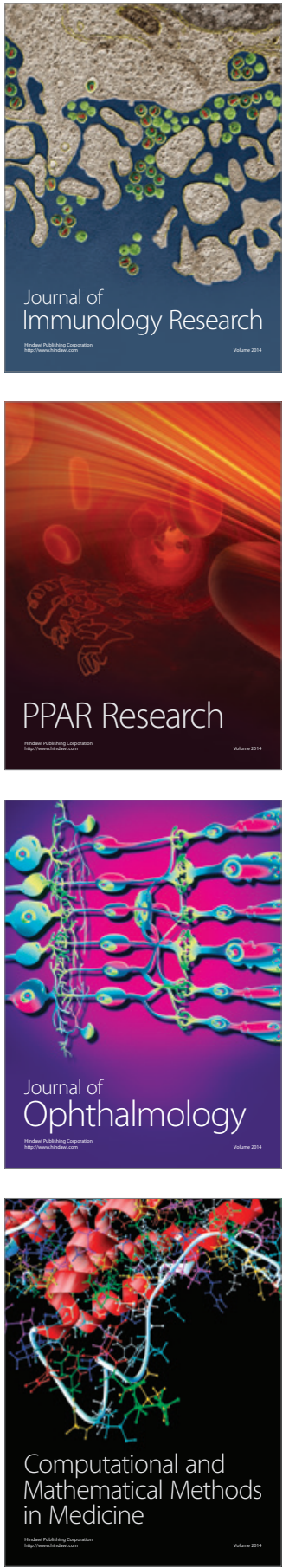

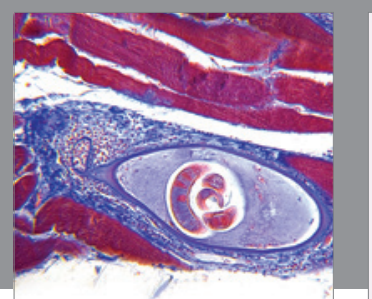

Gastroenterology Research and Practice

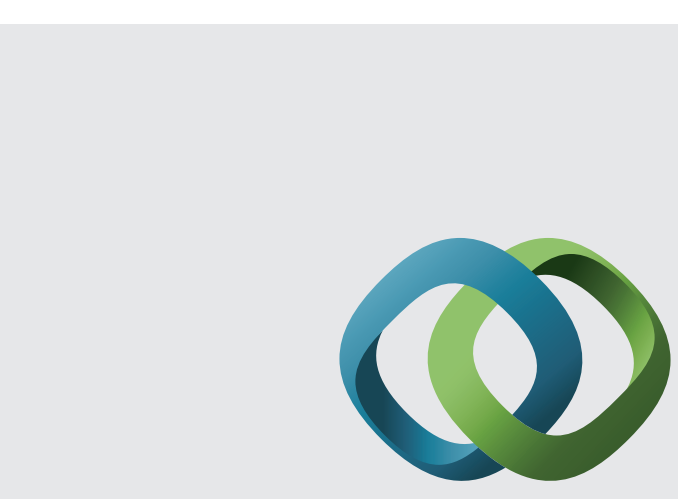

\section{Hindawi}

Submit your manuscripts at

http://www.hindawi.com
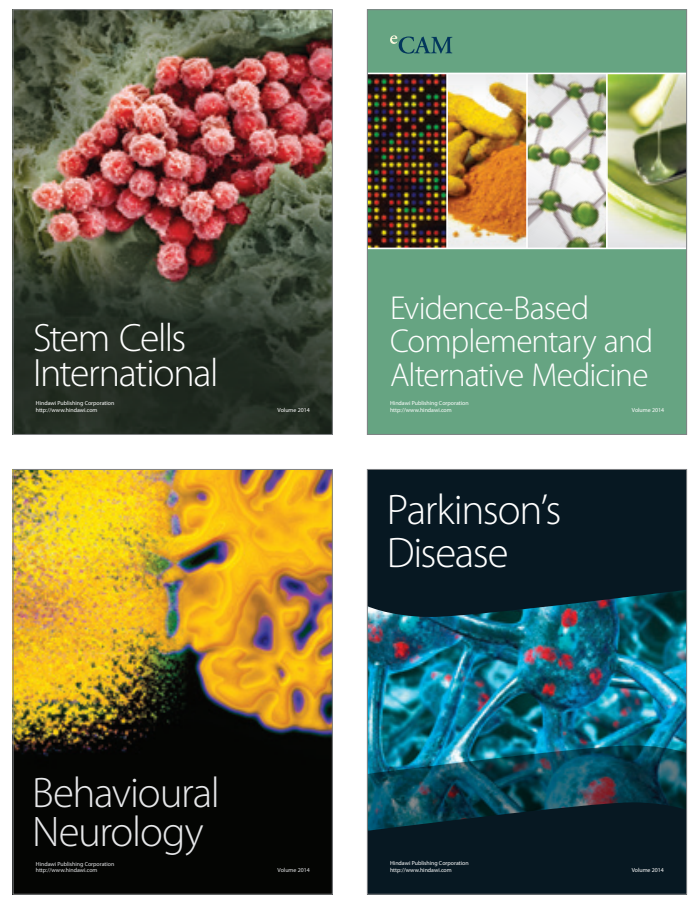
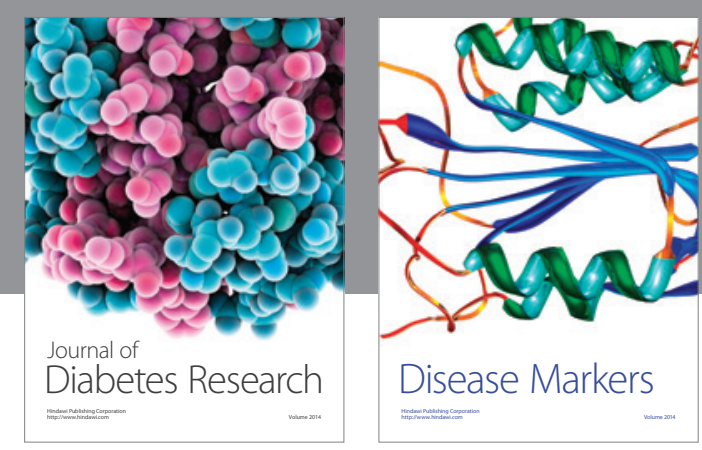

Disease Markers
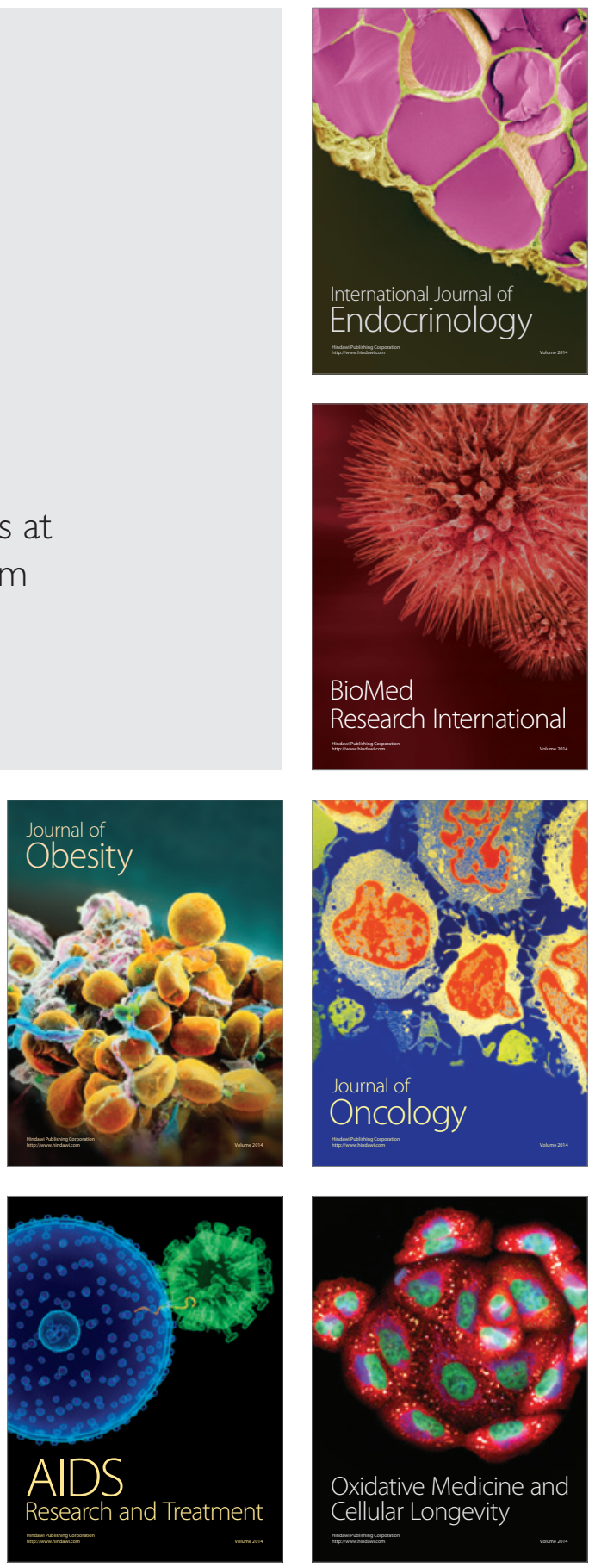